\title{
Adaptive sidelobe control for clutter rejection of atmospheric radars
}

\author{
K. Kamio ${ }^{1}{ }^{*}$, K. Nishimura ${ }^{1}$, and T. Sato ${ }^{1}$ \\ ${ }^{1}$ Kyoto University, Kyoto, Japan \\ *now at: Sony Corporation, Tokyo, Japan
}

Received: 1 September 2003 - Revised: 5 March 2004 - Accepted: 5 May 2004 - Published: 29 November 2004

Part of Special Issue "10th International Workshop on Technical and Scientific Aspects of MST Radar (MST10)"

\begin{abstract}
Clutter rejection is among the most important issues in radar signal processing, for which the adaptive antenna technique can be a powerful means. Compared to other applications of the adaptive antenna, however, atmospheric radars require strict conditions, which have prevented application of this technique; the main antenna beam pattern should not be altered since the target region is defined by its shape. In particular, the loss of the antenna gain should be kept to no more than about $0.5 \mathrm{~dB}$, in order to maintain the high sensitivity of the system. Also, clutter from surrounding mountains is often stronger than the desired weak scattering from atmospheric turbulence. We introduce a new algorithm which satisfies the above conditions, and confirms its capability by applying it to actual data taken by the MU radar. This paper presents the first report that demonstrates the effectiveness of the adaptive antenna technique in atmospheric radar applications. Despite the fact that no information is given on the spectral features of the desired and undesired signals, only the clutter echoes from surrounding mountains were effectively cancelled without affecting the desired echoes from atmospheric turbulence.
\end{abstract}

Key words. Meteorology and atmospheric dynamics (instruments and techniques) - Radio science (signal processing)

\section{Introduction}

Sidelobe cancelling or in a more general sense, the adaptive antenna technique, has been an important issue in various fields of antenna engineering. It is now gathering wide attention since the speed of signal processing devices has reached the level that enables real-time processing required to practice the theory.

In atmospheric radar applications, where a sharp antenna beam is usually configured by a large array or aperture

Correspondence to: T. Sato

(tsato@kuee.kyoto-u.ac.jp) antenna, strong clutter echoes from surrounding mountains are the major source of interference. As the desired echoes from the atmosphere are so weak, even very weak echoes entering low-level sidelobes can become a serious problem especially when they contain fluctuating components (Sato and Woodman, 1982). It has been a common practice to remove such undesired echoes in the off-line signal processing after the data is recorded.

A clear advantage of the adaptive antenna is that it makes use of extra information about the direction of arrival in discriminating undesired echoes, in contrast to other clutter rejection schemes based on signal processing of the received echoes, such as adaptive filtering. As the adaptive filtering technique tries to remove the clutter after it is mixed with the desired echo, it cannot completely remove the clutter when the clutter has a fading component. It also tends to "overkill" the DC component of the desired echo, as is the case in observing the vertical direction. It is thus quite effective to cancel the clutter echo before it is mixed with the desired echo by modifying the antenna pattern adaptively.

However, conventional algorithms for the adaptive antenna have the serious defect of distorting the antenna's main beam pattern when they are directly applied to atmospheric radars. The original sidelobe canceller by Howells (1965) may cancel the output of the main beam under extremely strong interference signal. This effect is mitigated by adding a limiter in its feedback loop (Abe et al., 1995), but the threshold should be controlled according to the interference level. Since the sidelobe canceller is regarded as a type of adaptive antenna, the Directionally Constrained Minimum Power (DCMP) algorithm (Takao et al., 1976) can be used to suppress the sidelobe echoes while maintaining the desired signal. The same principle is also known as Minimum Variance Distortionless Response (MVDR) (Haykin, 2001). This idea is further utilized in a variety of Generalized Sidelobe Canceling (GSC) algorithms (Griffiths and Jim, 1982), which assure the response in the desired direction by controlling the weight of an output which does not contain the desired signal. Efforts have been made to effectively delete the 
desired signal in this output by means of block filters (Fudge and Linebarger, 1996; Chu and Fang, 1999; Wang and Fang, 2000).

Performance of these algorithms depend on the characteristics of the desired signal, and the shape of the main beam may be altered when the cancellation is not complete. The main beam pattern is an essential design factor in the atmospheric radars, since the target is widely distributed in space, and the "desired signal" is defined as the echoes which return from the main lobe region. Even a slight change in the shape of the main beam will result in an offset of the estimated wind velocity.

Here we introduce a new sidelobe cancelling algorithm (Kamio and Sato, 2004), which extends DCMP by introducing an additional constraint on the weight of the receiving array so that the main beam pattern of the radar is conserved. We demonstrate its effectiveness by applying the technique to actual data taken with the MU (Middle and Upper atmosphere) radar as the first attempt to introduce the adaptive antenna technique to atmospheric radar. Its performance and limitations for various types of clutters are examined with actual data, as well as numerical simulations. We further examine the feasibility of rejecting non-stationary clutter, such as reflection echoes from aircraft.

\section{Proposed algorithm}

A received signal of a phased array is given by

$y=\boldsymbol{W}^{H} \boldsymbol{X}$,

where $\boldsymbol{X}$ and $\boldsymbol{W}$ are the complex input signal vector and the weight vector, respectively. The output power is expressed in terms of the covariance matrix $\mathbf{R}_{\mathbf{x x}}$ as

$P=\frac{1}{2}\left[|y|^{2}\right]=\frac{1}{2} \boldsymbol{W}^{H} \boldsymbol{X} \boldsymbol{X}^{H} \boldsymbol{W}=\frac{1}{2} \boldsymbol{W}^{H} \mathbf{R}_{\mathbf{x x}} \boldsymbol{W}$.

The principle of DCMP algorithm (Takao et al., 1976) is to minimize the output power under the constraint

$\boldsymbol{W}^{H} \boldsymbol{C}=H^{*}$,

where $\boldsymbol{C}$ is the desired direction vector, and $H$ is the constraint. Here we further apply an alternate condition

$\boldsymbol{W}^{H} \boldsymbol{W} \leq U$,

which forces that the norm of the weight should be less than a given value $U$, which is set to be sufficiently lower than the main lobe level, but not so low as to affect the weight control of the sidelobe region. This second constraint assures that the entire main lobe pattern is not affected by the weight control. Here we call this algorithm as "DCMP Constrained Norm" (DCMP-CN), in contrast to conventional DCMP.

The principle of DCMP-CN is thus expressed as

${ }^{\min }\left(P_{\text {out }}=\frac{1}{2} \boldsymbol{W}^{H} \mathbf{R}_{\mathbf{x x}} \boldsymbol{W}\right)$

subject to $\boldsymbol{C}^{T} \boldsymbol{W}^{*}=H \& \boldsymbol{W}^{H} \boldsymbol{W} \leq U$.
This minimization problem with an equality constraint and an inequality condition is solved by using the penalty function method. The cost function is expressed as

$$
Q_{k}(x)=f(x)+\rho_{k}\left(\sum_{i=1}^{r}\left(g_{i}(x)\right)^{2}+\sum_{r+1}^{m}\left(g_{i}(x)\right)_{-}{ }^{2}\right),
$$

where $f(x)$ is the function to be minimized, $g_{i}(x)=0$ gives an equality constraint, and $g_{i}(x)_{-}=0$ gives an inequality constraint. Here $(a)_{-}=\min \{0, a\}=(a-|a|) / 2, r$ is the number of equality constraints, and $(m-r)$ is the number of inequality constraints.

We choose an arbitrary increasing series $\left\{\rho_{k}\right\}$ of the penalty factor which goes to infinity with increasing $k$. For each $k$, we minimize $Q_{k}(x)$ with a nonlinear, unconstrained optimization algorithm to obtain $x_{k}$ starting from $x_{k-1}$. In actual processing, we set $\rho_{0}$ to the received signal power of a subarray element with a randomly chosen weight value within the limit of the norm constraint for $U$, and let $\rho_{k}=10 \rho_{k-1}$ for $k=1, \ldots, 4$. We terminate the iteration at $k=4$, where we obtain a sufficiently stable solution.

The cost function for the current case is given by

$$
\begin{aligned}
Q_{k}(\boldsymbol{W})= & \frac{1}{2} \boldsymbol{W}^{H} \mathbf{R}_{\mathbf{x x}} \boldsymbol{W}+\rho_{k}\left[\left|\boldsymbol{W}^{H} \boldsymbol{C}-H\right|^{2}\right. \\
& \left.+\left(U-\boldsymbol{W}^{H} \boldsymbol{W}\right)_{-}^{2}\right] \\
= & \frac{1}{2} \boldsymbol{W}^{H} \mathbf{R}_{\mathbf{x x}} \boldsymbol{W}+\rho_{k}\left[\left(\boldsymbol{W}^{H} \boldsymbol{C}-H\right)\left(\boldsymbol{C}^{H} \boldsymbol{W}-H^{*}\right)\right. \\
& \left.+\left(U-\boldsymbol{W}^{H} \boldsymbol{W}\right)_{-}{ }^{2}\right] .
\end{aligned}
$$

The gradient of $Q_{k}(\boldsymbol{W})$ in terms of the weight vector $\boldsymbol{W}$ is given by

$$
\begin{aligned}
\nabla_{w} Q_{k}(\boldsymbol{W})= & \mathbf{R}_{\mathbf{x x}} \boldsymbol{W}+\rho_{k}\left[2 \boldsymbol{C}\left(\boldsymbol{C}^{H} \boldsymbol{W}-H^{*}\right)\right. \\
& \left.-4 \boldsymbol{W}\left(U-\boldsymbol{W}^{H} \boldsymbol{W}\right)_{-}\right] .
\end{aligned}
$$

\section{Application to high gain arrays}

Here we consider the application of DCMP-CN to the case of a high-gain antenna array consisting of several hundred elements. In such a case, it is not practical to control all of the elements. Instead, we select several antennas at the outer edge of the array to configure a sub-array, and only control the weights of its elements, as shown in Fig. 1, keeping the weight of the main array output to 1 . In the radar application, the main array is used both for transmission and reception, and the sub-array is used only for reception. This configuration is useful in suppressing the clutter echoes of existing radar by adding several receiving antenna elements. In this case, the output power is rewritten as

$$
\begin{aligned}
P_{\text {out }}= & \frac{1}{2} \boldsymbol{W}^{H} \mathbf{R}_{\mathbf{x x}} \boldsymbol{W} \\
= & \frac{1}{2}\left(x_{1} x_{1}^{*}+\boldsymbol{W}_{2: n}^{H} \boldsymbol{X}_{2: n} x_{1}^{*}+x_{1} \boldsymbol{X}_{2: n}^{H} \boldsymbol{W}_{2: n}\right. \\
& \left.+\boldsymbol{W}_{2: n}^{H} \tilde{\mathbf{R}}_{\mathbf{x x}} \boldsymbol{W}_{2: n}\right),
\end{aligned}
$$




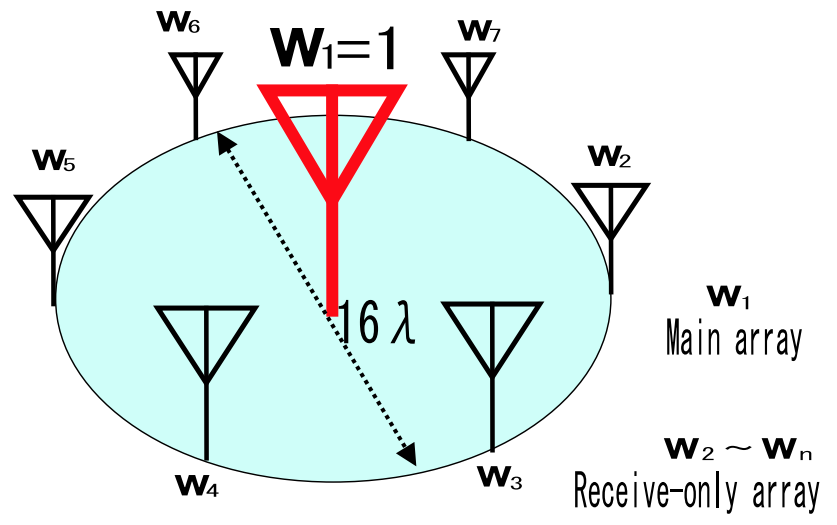

Fig. 1. Configuration of a high-gain antenna with a peripheral receive-only sub-array.

where subscript 1 denotes the output of the main array, and 2 to $n$ correspond to the sub-array. Since the constraints are given only to the sub-array elements, the problem is expressed as

$$
\begin{aligned}
& \min _{\boldsymbol{W}}\left(P_{\text {out }}=\frac{1}{2}\left(x_{1} x_{1}^{*}+\boldsymbol{W}_{2: n}^{H} \boldsymbol{X}_{2: n} x_{1}^{*}+x_{1} \boldsymbol{X}_{2: n}^{H} \boldsymbol{W}_{2: n}\right.\right. \\
& \left.+\boldsymbol{W}_{2: n}^{H} \tilde{\mathbf{R}}_{\mathbf{x} \mathbf{x}} \boldsymbol{W}_{2: n}\right) \\
& \text { subject to } C_{2: n}^{T} \boldsymbol{W}_{2: n}^{*}=H \& \boldsymbol{W}_{2: n}^{H} \boldsymbol{W}_{2: n} \leq U .
\end{aligned}
$$

The cost function is then given by

$$
\begin{aligned}
Q_{k}(\boldsymbol{W})= & \frac{1}{2}\left(x_{1} x_{1}^{*}+\boldsymbol{W}_{2: n}^{H} \boldsymbol{X}_{2: n} x_{1}^{*}\right. \\
& \left.+x_{1} \boldsymbol{X}_{2: n}^{H} \boldsymbol{W}_{2: n}+\boldsymbol{W}_{2: n}^{H} \tilde{\mathbf{R}_{\mathbf{x}}} \boldsymbol{W}_{2: n}\right) \\
& +\rho_{k}\left[\left(\boldsymbol{W}_{2: n}^{H} \boldsymbol{C}_{2: n}-H\right)\left(\boldsymbol{C}_{2: n}^{H} \boldsymbol{W}_{2: n}-H^{*}\right)\right. \\
& \left.+\left(U-\boldsymbol{W}_{2: n}^{H} \boldsymbol{W}_{2: n}\right)_{-}^{2}\right] .
\end{aligned}
$$

\section{Observations}

We applied this algorithm to the data taken with the MU (Middle and Upper Atmosphere) radar. It is a large atmospheric radar with a flexible active phased array antenna consisting of 475 Yagi-Uda antennas (Fukao et al., 1985a,b). Its main parameters are summarized in Table 1.

The antenna array consists of 25 groups of hexagonal subarray with 19 crossed 3-element Yagi antennas. A transmit/receive module is connected to each Yagi antenna. On reception, the RF signal at $46.5 \mathrm{MHz}$ is converted to IF of $5 \mathrm{MHz}$ at each module, and the output of 19 modules are combined at each group. Combined IF signals from 25 groups are sent to the control building, and divided for 4 receiver channels. Each receiver can select and combine output

\begin{tabular}{|c|c|}
\hline Parameter & Value \\
\hline Location & $\begin{array}{l}\text { Shigaraki, Shiga, Japan } \\
\left(34.85^{\circ} \mathrm{N}, 136.10^{\circ} \mathrm{E}\right)\end{array}$ \\
\hline Radar system & $\begin{array}{l}\text { monostatic pulse radar; } \\
\text { active phased array system }\end{array}$ \\
\hline Frequency & $46.5 \mathrm{MHz}$ \\
\hline Antenna & circular array of 475 crossed Yagi's \\
\hline $\begin{array}{l}\text { beam width } \\
\text { steerability } \\
\text { beam directions } \\
\text { polarizations }\end{array}$ & $\begin{array}{l}3.6^{\circ} \text { (one way; half power for full array) } \\
\text { steering is completed in each IPP } \\
1657 ; 0^{\circ}-30^{\circ} \text { off zenith angle } \\
\text { linear and circular }\end{array}$ \\
\hline Transmitter & 475 solid state amplifiers \\
\hline peak power & $1 \mathrm{MW}$ (maximum) \\
\hline average power & $50 \mathrm{~kW}$ (duty ratio $5 \%$ ) (maximum) \\
\hline bandwidth & $\begin{array}{l}1.65 \mathrm{MHz} \text { (maximum) } \\
\quad \text { (pulse width: } 1-512 \mu \text { s variable) }\end{array}$ \\
\hline Range resolution & $150 \mathrm{~m}$ \\
\hline
\end{tabular}
from 25 groups at an arbitrary selection. Figure 2 shows the outline of the signal processing system.

We conducted an experiment making use of this flexibility. Output from all groups except for 3 groups at the outer edge of the array is fed to a receiver, as shown in Fig. 3. For the
Table 1. Basic parameter of the MU radar.

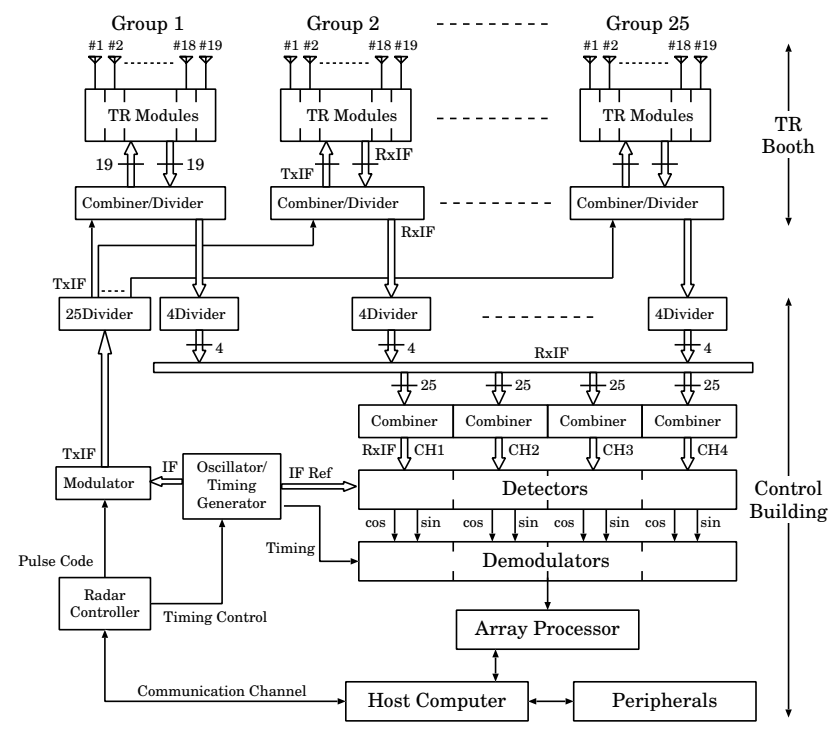

Fig. 2. Receiver and signal processing system at the MU radar.

rest of the 3 groups, only one antenna is activated in each group, and connected to three receivers.

Observation was made for about two hours from 06:4507:56 JST and 17:18-18:21 JST on 26 December 2002, with $1-\mu \mathrm{s}$ pulse transmissions at $400 \mu \mathrm{s}$ intervals. The antenna beam was tilted $10^{\circ}$ from the zenith, and tropospheric echoes were sampled from $1.5 \mathrm{~km}$ to $9.6 \mathrm{~km}$ height region at $150-\mathrm{m}$ sampling intervals. The received time series was averaged over 19 pulses for each range gate and recorded for off-line processing. 


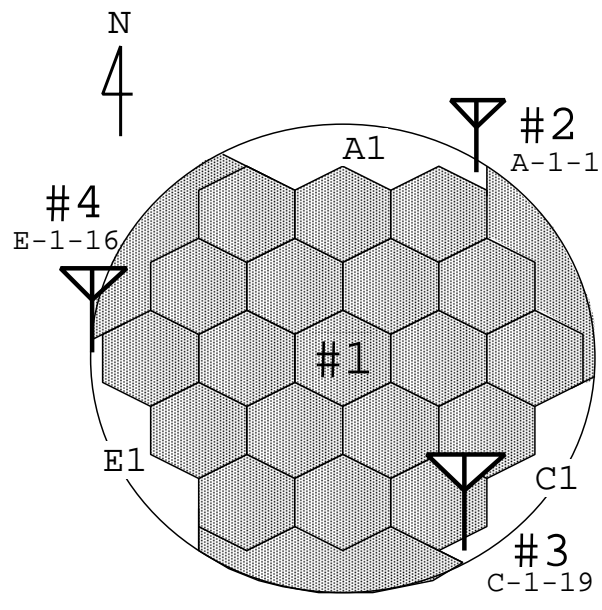

Fig. 3. Antenna field configuration and antenna positions for the observation in this paper.

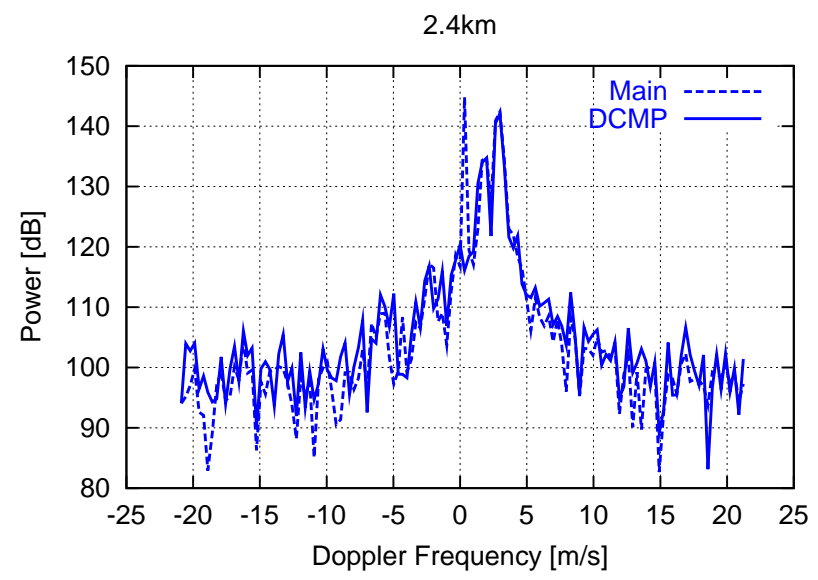

Fig. 4. Doppler echo power spectrum at $2.4 \mathrm{~km}$ range raw main beam data (dashed) and processed with conventional DCMP algorithm (solid). The y-axis indicates received power in arbitrary units.

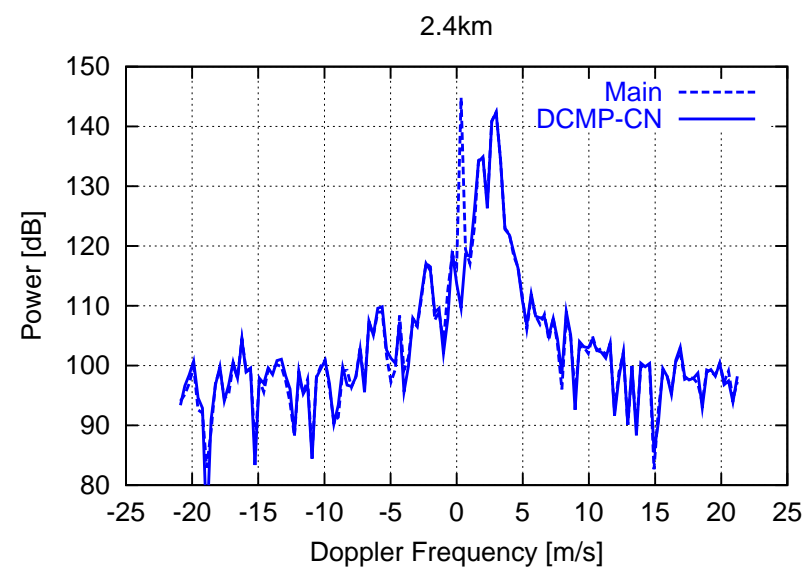

Fig. 5. Doppler echo power spectrum at $2.4 \mathrm{~km}$ range raw main beam data (dashed) and processed with the proposed DCMP-CN algorithm (solid).

\section{Clutter suppression using real data}

Here we use the above data to examine the effectiveness of our proposed algorithm. The entire antenna array is regarded as the main antenna, and three antennas connected to the other three receivers constitute the sub-array. The weight of the main channel is fixed, and the complex weight of the output from the other three channels are varied so that the clutter echo is suppressed. By controlling the weight of only the sub-array, complexity of the adaptive processing is drastically reduced. Also, this system can be easily applied to existing radar systems.

The maximum constraint of the weight norm $U$ is set to 0.5 . In generating the covariance matrix $\mathbf{R}_{\mathbf{x x}}$, instantaneous samples are averaged with a decay factor of $\beta=0.997$, which is roughly equivalent to taking the average of 1000 samples. The appropriate values of $U$ and $\beta$ are discussed in more detail in Sects. 6 and 7, respectively.

Figure 4 shows an example of the echo power spectrum. The spectrum was generated by applying 128-point FFT to a time series of $9.7 \mathrm{~s}$ after coherent integration of 190 pulse samples. No incoherent integration is applied. The vertical scale is in an arbitrary unit, and the galactic background noise level is about $100 \mathrm{~dB}$. The dashed line shows the original spectrum with the main antenna. The sharp spike at zero Doppler velocity is the clutter component, while a broad peak with positive Doppler shift is the desired echo. The solid line shows the result of conventional DCMP, with which the clutter is suppressed with a penalty of increased noise level.

Figure 5 shows the same spectrum processed with the proposed DCMP-CN algorithm. The solid line exactly follows the desired signal component by rejecting the clutter component only. It should be noted that we assume no information on the echo power spectrum, such as the narrow clutter spectrum. It is therefore possible to cancel not only the DC clutter, but also clutters with fading as far as their time constant is long enough compared to the time required to obtain the covariance matrix.

The increased noise level with the conventional algorithm is due to the large weight of the sub-array elements, which enhanced the galactic noise level and also atmospheric echoes entering low elevation sidelobes, which spreads out in a broad Doppler spectrum. On the other hand, the proposed DCMP-CN algorithm effectively suppresses such an increase by controlling the weight of the sub-array elements. This example clearly demonstrates the usefulness of adaptively controlling the antenna pattern with a sub-array configuration.

It should be noted that the adaptive cancellation needs to be applied separately for different range gates and beam directions. In this manner, clutters from various directions can be suppressed with a small number of sub-array elements, as long as they have a different range from the radar. As the computational load of the proposed algorithm is very light, more than 1000 time series corresponding to different range gates and beam directions can be handled simultaneously with a personal computer. 


\section{Considerations on the constraints}

In the previous section, we set the maximum norm constraint $U$ to 0.5 . Here we examine the appropriate range of this value, and also the relative importance of the norm constraint over the directional constraint.

The proposed DCMP-CN algorithm has the advantage that the control parameter $U$ can be chosen regardless of the strength of the desired and undesired signals, since it is determined simply by the relative pattern of the main and subarray antennas. However, there is a certain range of $U$ that gives the best performance.

If we set $U$ to a value that is too large, the DCMP-CN algorithm approaches to DCMP, and thus becomes unable to suppress the increase in the noise level, as shown in Fig. 4. On the other hand, if we set $U$ to a value that is too small, the algorithm may not be able to cancel the clutter component by the combined output of sub-array elements.

We first consider a case where the output of a sub-array element is added to that of the main array consisting of $M$ elements. We assume that the sub-array element is the same as that of the main array, and the output is in phase for the main beam direction. Also, in Eq. (10) we implicitly assumed that the output of the sub-array element is normalized to that of the main array at the isotropic-gain level. In the VHF range, where the noise is dominated by the galactic background radiation, it is equivalent to normalize them by their noise level.

In this case, the signal power after adding the sub-array element increases by $(\sqrt{M}+1)^{2} / M$ times, while the noise level increases by a factor of 2 . The gain of the signal-tonoise ratio by adding a sub-array element is thus given by

$G_{\mathrm{SNR}}=\frac{(\sqrt{M}+1)^{2}}{2 M}$,

which is roughly $-3 \mathrm{~dB}$ for a large $M$.

Next we consider a more general case where the sub-array element has a relative gain of $G_{\mathrm{s}}$ in the main beam direction of the main-array element, the output power of the sub-array element is multiplied by $U$ before addition, and it may not be in phase with the main array. In this case, Eq. (13) becomes

$G_{\mathrm{SNR}}=\frac{\left(\sqrt{M}+\alpha \sqrt{G_{\mathrm{s}} U}\right)^{2}}{(1+U) M}$,

where $\alpha$ takes a value between -1 and 1 , and is 1 for the case where two signals are in phase, 0 for the orthogonal phase, and -1 for the opposite phase. This situation corresponds to the worst possible case of the proposed algorithm with a norm constraint value of $U$. Although we have examined only the case of one antenna element, Eq. (14) can also be applied to a sub-array consisting of multiple antenna elements, because the norm constraint $U$ limits the sum of the weight of the sub-array elements.

In the case shown in Fig. 5, $G_{\mathrm{s}}=1(0 \mathrm{~dB})$ and $U=0.5$, which gives $G_{\mathrm{SNR}}$ of $-1.5 \mathrm{~dB}$. However, the actual loss is much less than this value, as is clear from the figure, because

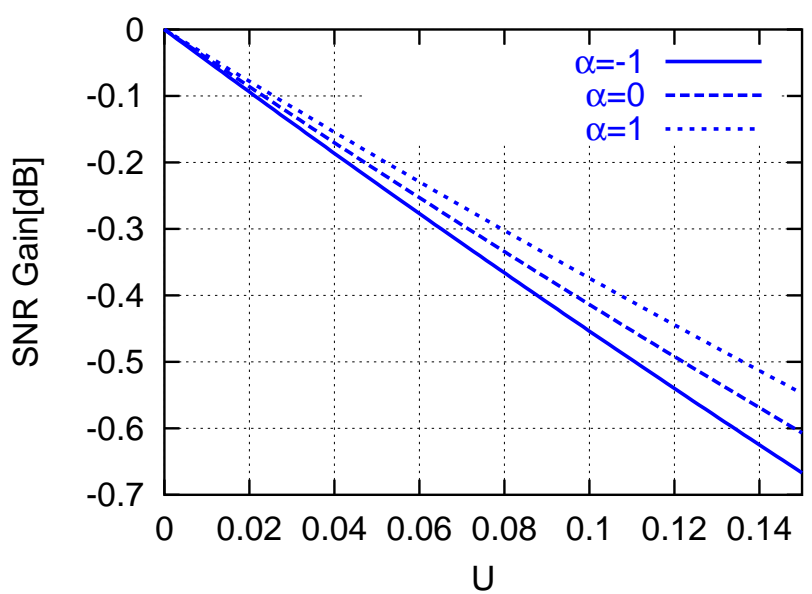

Fig. 6. SNR loss as a function of constrained norm value $U$.

Eq. (14) gives the worst case. Figure 6 shows $G_{\text {SNR }}$ versus $U$ for the case of $M=475$, assuming the MU radar, and $G_{\mathrm{s}}=U$.

Apparently, a small constraint is desirable in order to assure a small loss in SNR. If we allow a loss of up to $0.5 \mathrm{~dB}$, the norm constraint $U$ should be set to $0.135,0.120$, and 0.105 for the in-phase, orthogonal, and the out-of-phase cases, respectively. As we assumed that the antenna element used for the sub-array has a gain of $G_{\mathrm{s}}=U$ relative to that for the main-array element, it means that the sub-array element should have a relative gain of $-9.2 \mathrm{~dB}$ in the main lobe direction for $U=0.12$. In the case of the MU radar, as a typical example of VHF MST radars, 3-element Yagi antenna elements with an isotropic gain of $7.2 \mathrm{~dB}$ are pointed to the zenith, and the main lobe is steered in an angular region of $30^{\circ}$ from the zenith. An isotropic gain of less than $-2 \mathrm{~dB}$ is easily achieved in this angular region by pointing the same element to the horizontal direction, for example. If a specially designed antenna element which has less sensitivity to the main lobe region is used for the sub-array element, a larger value of $U$ becomes acceptable.

If we apply the proposed DCMP-CN algorithm, the subarray elements should always be kept in phase with the main antenna because of the directional constraint. The orthogonal and the out-of-phase cases examined above correspond to situations where this constraint is not applied. Figure 6 shows that if we remove the directional constraint from the algorithm with $U=0.12$, we further lose about $0.13 \mathrm{~dB}$, or $3 \%$, of the sensitivity. The advantage of not applying the directional constraint is that a simpler algorithm with only the norm constraint can be used, and that no phase calibration is required for the sub-array elements.

\section{Suppression of clutter from moving targets}

The example shown in the previous section clearly demonstrates the usefulness of the proposed method in rejecting the clutter from stationary targets. As we mentioned, the same 


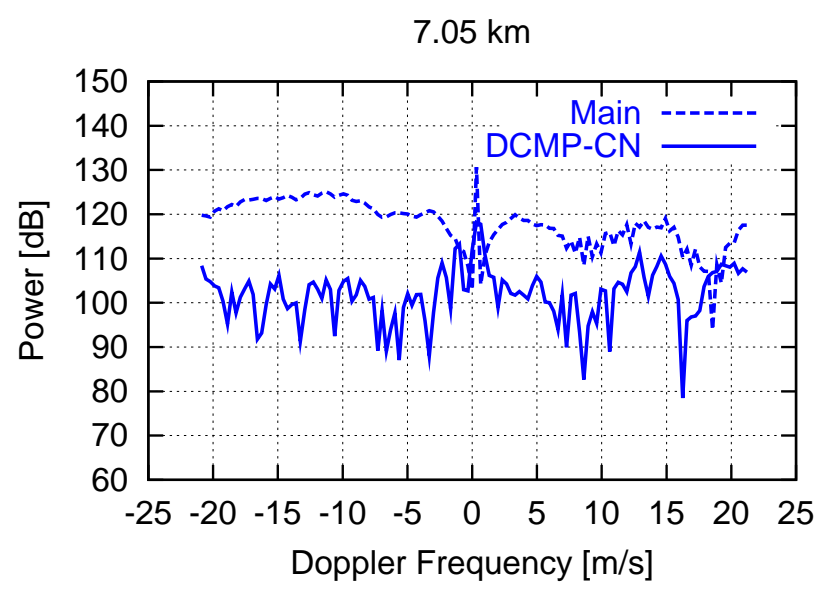

Fig. 7. Example of the Doppler echo power spectrum with strong aircraft echo at a sampling interval of $76 \mathrm{~ms}$ from raw main beam data (dashed) and with proposed DCMP-CN algorithm (solid). The $\mathrm{y}$-axis indicates received power in arbitrary units.

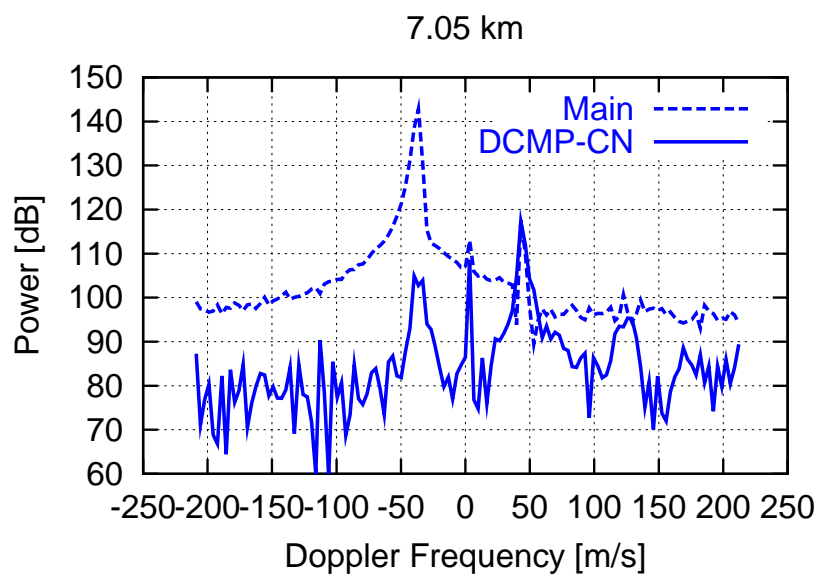

Fig. 8. Same as Fig. 7, but for a sampling interval of $7.6 \mathrm{~ms}$.

algorithm can be applied without any problem to the cases where the undesired echoes are fading, as long as they have no correlation with the desired echo. However, if the direction of arrival of the undesired echoes change in time, as is the case for the clutter echoes from aircraft or the coherent echoes from ionospheric irregularities, for example, the algorithm has to catch up with this change by modifying the antenna pattern. This limits the time that can be used to average the covariance matrix $\mathbf{R}_{\mathbf{x x}}$.

Here we examine a case of aircraft echo, which is the most typical undesired echo with rapid motion for the MST radar environment. As for the MU radar, the major source of aircraft clutter is regular customer jet planes, whose closest route is about $7 \mathrm{~km}$ from the radar site. Although the aircraft echoes are received through low elevation sidelobes, they are much stronger than the desired weak scattering from atmospheric turbulence.

If we consider a jet plane flying on a linear route whose minimum distance from the radar is $7 \mathrm{~km}$ at a speed of
$280 \mathrm{~m} / \mathrm{s}$, its maximum angular motion is $2.3^{\circ} / \mathrm{s}$ at its closest point. Figure 7 shows an example of the echo power spectrum with an aircraft at its closest range of $7.05 \mathrm{~km}$. The received data are first coherently averaged for 190 pulses $(=76 \mathrm{~ms})$, and the power spectrum is computed by using 128-point FFT, then incoherently averaged for 10 times $(=7.6 \mathrm{~s})$, as is the case for Figs. 4 and 5. The atmospheric echo was below the noise level on this day at this range, so only the clutter echoes from the aircraft and mountains are present. The dashed line is the original power spectrum observed by the main antenna. As the Doppler velocity of the aircraft echo changes from $+42 \mathrm{~m} / \mathrm{s}$ to $-42 \mathrm{~m} / \mathrm{s}$ in an interval of $7.6 \mathrm{~s}$ around its closest point, its echo power spreads over the entire spectral window.

The solid line is after processing by the proposed algorithm with the sub-array signals. In this case, the covariance matrix $\mathbf{R}_{\mathbf{x x}}$ was averaged with the decay factor $\beta=0.7$, which corresponds to an averaging over only about 10 data samples compared to about 1000 samples used in the previous section, in order to adjust to the rapid motion of the target. The stationary clutter component at 0 Doppler shift is suppressed by $12 \mathrm{~dB}$, and the aircraft echo is suppressed by about $15 \mathrm{~dB}$. The small reduction in the stationary clutter component is apparently due to the small number of samples averaged to form the covariance matrix. The suppression of the moving clutter component is also limited, but has a substantial effect in estimating one-hour mean wind profiles, for example, because the remaining clutter component can be further suppressed by incoherent integration over a one-hour period.

One possible means of further reducing the aircraft echoes is to employ faster sampling. Figure 8 is the same as Fig. 7, except that the coherent integration time is reduced to 19 times instead of 190 . The decay factor and the number of FFT is kept the same, so everything is computed based on 10 times faster sampling and shorter duration. It should be noted that the maximum Doppler velocity is thus 10 times larger, and the power level is $20 \mathrm{~dB}$ lower because the number of coherent addition is $1 / 10$.

As the Doppler velocity of the aircraft changes only by $8.4 \mathrm{~m} / \mathrm{s}$ during the $0.76 \mathrm{~s}$ period used for computation, its echo appears as a clear spike at around $-40 \mathrm{~m} / \mathrm{s}$. The peak at $+40 \mathrm{~m} / \mathrm{s}$ is its spectral image due to a gain imbalance of the two orthogonal channels of the receiver. The aircraft echo is reduced by about $40 \mathrm{~dB}$ after the adaptive suppression, as shown by the solid line. Since the spectral image component is generated inside the receiver, it cannot be cancelled by the adaptive algorithm, which naturally assumes an ideal receiver and generates a pure signal without the image in cancelling the clutter.

The reason that the stationary clutter from mountains is even less suppressed compared to Fig. 7 is probably that the relative magnitude of the mountain echo to the aircraft echo is smaller than the previous case, and the number of subarray elements $(=3)$ is not large enough to cancel this weak interference. 


\section{Averaging of the covariance matrix}

The examples presented in the previous section clearly show the effectiveness of reducing the time used for adaptation in order to suppress clutter echoes from a moving target. On the other hand, reducing the degree of averaging in estimating the covariance matrix $\mathbf{R}_{\mathbf{x x}}$ causes an inaccurate estimation of the signal characteristics, which results in an incomplete cancellation of undesired signals.

Here we examine the effect of the degree of averaging on the performance of the algorithm via numerical simulations. We assume a sub-array consisting of three elements as the actual observation presented in this paper. The main beam direction is $10^{\circ}$ from the zenith, and a stationary clutter arrives from zenith angle of $75^{\circ}$. The echo power of the desired and interference signals are set to $0 \mathrm{~dB}$ and $70 \mathrm{~dB}$, respectively. The main beam has a one-way gain of $34 \mathrm{~dB}$.

As the accuracy of estimating the covariance matrix will be strongly affected by the magnitude of phase rotation of the signal due to the Doppler effect, we consider three cases of the line-of-sight Doppler velocity of $0.0 \mathrm{~m} / \mathrm{s}, 0.33 \mathrm{~m} / \mathrm{s}$, and $2.3 \mathrm{~m} / \mathrm{s}$. The signal is coherently added for 190 pulse samples, or $76 \mathrm{~ms}$, before processing. Figure 9 shows the depth of the null in the direction of the clutter, assuming that the desired signal and the clutter are pure sinusoid.

For the case of $0.0 \mathrm{~m} / \mathrm{s}$, there is no means to discriminate the desired signal from the clutter because they are both sinusoid of the same frequency. The algorithm thus cancels the clutter by controlling its amplitude equal to that of the desired signal, and with opposite phase. As the main beam has a gain of $34 \mathrm{~dB}$, the undesired clutter component with a $70 \mathrm{~dB}$ higher level is cancelled by controlling the antenna gain to this direction, to $-36 \mathrm{~dB}$, as shown in this figure. Apparently, a larger suppression is required in order to detect the desired signal.

The suppression rapidly increases as the Doppler velocity and the degree of averaging increases. The periodicity found in other cases than zero Doppler agrees with the period of the given phase rotation of the signal. It is found that the number of phase rotation needed to obtain the SIR (Signalto-Interference Ratio) of $25 \mathrm{~dB}, 30 \mathrm{~dB}$, and $35 \mathrm{~dB}$ during the averaging period is about 5 cycles, 9 cycles, and 17 cycles, respectively.

Since the actual atmospheric echo has a finite spectral width, we also examined such cases. Figure 10 is the same as Fig. 9, but the signal has a Gaussian spectrum with the halfpower width of $0.33 \mathrm{~m} / \mathrm{s}$. The suppression for both $0.33 \mathrm{~m} / \mathrm{s}$ and $2.33 \mathrm{~m} / \mathrm{s}$ cases are roughly equal to those cases in Fig. 9, indicating that the mean phase rotation is more important than random phase changes. For the case of zero Doppler shift, however, a steady increase in suppression is found as the degree of averaging increases. This is the effect of the random phase variation. Figure 11 shows the depth of the null for zero mean Doppler velocity and various spectral width. This situation is encountered when the antenna beam is pointed to the zenith, in order to observe the vertical wind velocity. It is thus important that the clutter echoes from

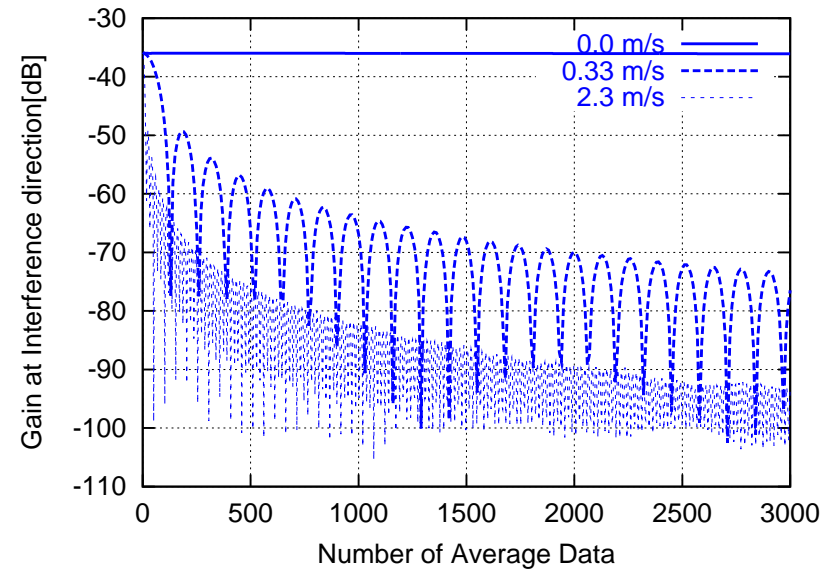

Fig. 9. Gain in direction of interferer for monochromatic signals with different Doppler velocities.

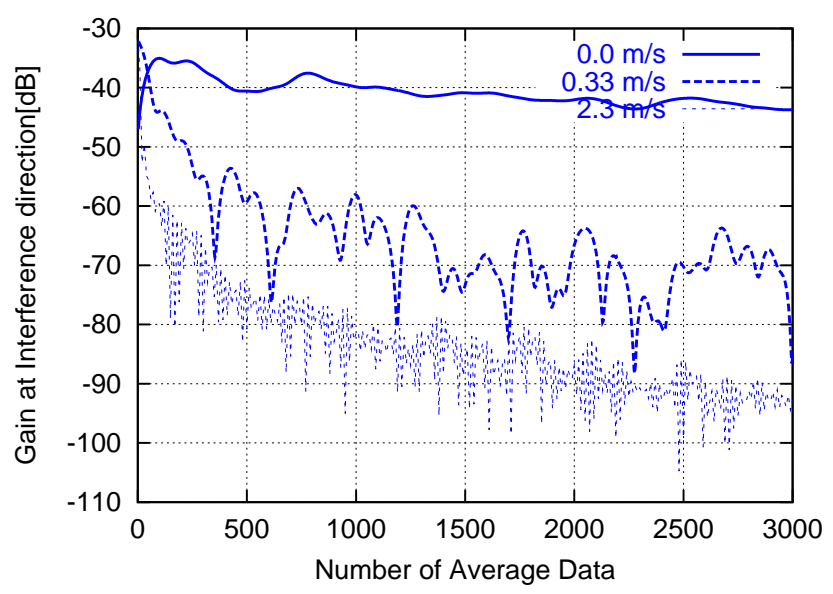

Fig. 10. Same as Fig. 9, but for the interferer with spectral width of $0.33 \mathrm{~m} / \mathrm{s}$.

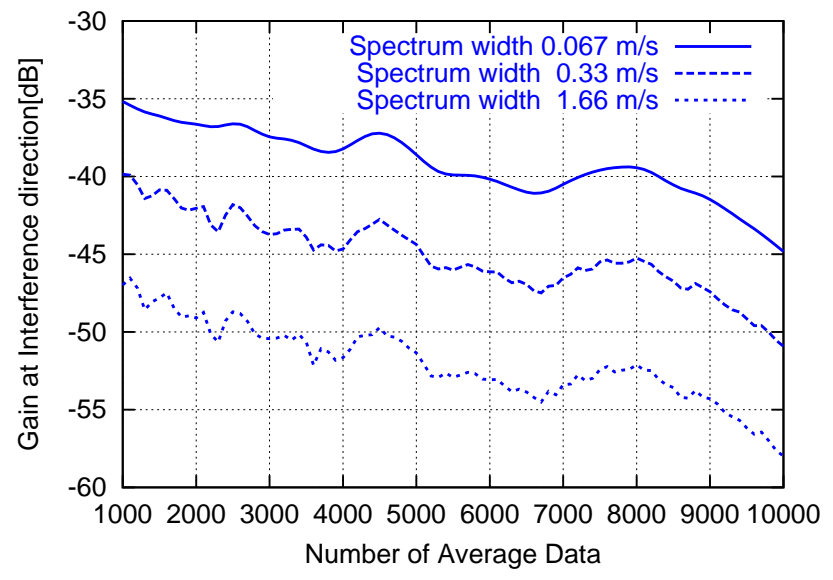

Fig. 11. Gain in direction of interferer for signals with different spectral width when no mean motion is present. 
mountains, which overlaps with the atmospheric echo power spectrum, can be suppressed by this method. For a typical spectral width of $0.33 \mathrm{~m} / \mathrm{s}$, SIR of $15 \mathrm{~dB}$ can be obtained by averaging for 10000 times, or $12.7 \mathrm{~min}$. This integration time is not a problem for stationary targets as mountains, but is clearly not consistent with the requirements for suppression of aircraft echoes. We need to develop algorithms which can deal with these different types of interferences simultaneously. A promising method will be to estimate the direction of arrival of mountain echoes when no aircraft echo is present, and then switches to a different mode when aircraft echo is detected. In the aircraft mode, an additional constraint to form a null in the fixed direction of mountain echo should be added together with adaptive cancellation of the aircraft echo by rapid updating of the covariance matrix.

\section{Summary}

In this paper, we applied an adaptive sidelobe suppression algorithm developed for a high-gain antenna to actual data taken by the MU radar as the first attempt to introduce the adaptive antenna technique to atmospheric radar, and confirmed its effectiveness in suppressing the clutter echoes from mountains.

By constraining the weight norm of the sub-array as well as the response of the main antenna to the desired direction, good cancellation of the undesired signal is achieved without disturbing the main beam pattern. It should be noted that the proposed algorithm does not require any knowledge on the input signal spectrum.

The proposed method can be easily implemented to existing high-gain antenna systems by adding a small number of receiving antenna elements and a personal computer which performs all necessary computation and control.

Considerations are made on the value of the norm constraint $U$ to achieve the best performance. One of the advantages of the proposed algorithm over other adaptive antenna algorithms is that the controlling parameter is independent of the strength of the interference signal, and depends only on the antenna pattern of the main antenna and the sub-array.

We further examined the possibility of suppressing the aircraft echoes, which rapidly change their direction of arrival. It is found that it is effective to reduce the time used for averaging the covariance matrix $\mathbf{R}_{\mathbf{x x}}$ in order to suppress such echoes. However, it contradicts the requirement for suppressing the mountain echoes under a condition of a small Doppler velocity of the atmospheric echo. A different approach is needed to simultaneously cope with these different types of interferences.

The effectiveness of the proposed algorithm should be further evaluated with larger data sets at various atmospheric radar situations. For this purpose, we are currently developing digital receive-only array systems to be implemented to the MU radar, and also to the Equatorial Atmosphere Radar in Indonesia.
Acknowledgements. The MU radar belongs to, and is operated by Research Institute for Sustainable Humanosphere, Kyoto University. Part of this study was supported by the Ministry of Education, Culture, Sports, Science and Technology through Grant-in-Aid for Scientific Research on Priority Areas, No. 13136205.

Topical Editor U.-P. Hoppe thanks T. Turunen and another referee for their help in evaluating this paper.

\section{References}

Abe, K., Hirasawa, K., and Watanabe, H.: Radar sidelobe canceller characteristics in high power interference, IEICE Trans. Commun., E78-B, 1507-1512, 1995.

Chu, Y. and Fang, W.: A novel wavelet-based generalized sidelobe canceller, IEEE Trans. Antennas Propagat., 47, 1485-1495, 1999.

Fudge, G. L. and Linebarger, D. A.: Spatial blocking filter derivative constraints for the generalized sidelobe canceller and MUSIC, IEEE Trans. Signal Processing, 44, 51-61, 1996.

Fukao, S., Sato, T., Tsuda, T., Kato, S., Wakasugi, K., and Makihira, T.: The MU radar with an active phased array system 1 . Antenna and power amplifiers, Radio Sci., 20, 1155-1168, 1985a.

Fukao, S., Tsuda, T., Sato, T., Kato, S., Wakasugi, K., and Makihira, T.: The MU radar with an active phased array system 2 . In-house equipment, Radio Sci., 20, 1169-1176, 1985 b.

Griffiths, L. J. and Jim, C. W.: An alternative approach to linearly constrained adaptive beamforming, IEEE Trans. Antennas Propagat., 30, 27-34, 1982.

Haykin, S. S.: Adaptive filter theory, 4th Ed., Prentice Hall, New York, 995, 2001.

Howells, P. W.: Intermediate frequency sidelobe canceller, U.S. Patent No. 3202990, 1965.

Kamio, K. and Sato, T.: An adaptive sidelobe cancellation algorithm for high-gain antenna arrays, Electron. Commun. in Japan, 87, 11-18, 2004.

McWhirter, J. G.: Data-domain penalty function algorithm for stabilized adaptive beamforming, IEE Proc. Radar, Sonar Navig., 147, 265-269, 2000.

Sato, T. and Woodman, R. F.: Spectral parameter estimation of CAT radar echoes in the presence of fading clutter, Radio Sci., 17, 817-826, 1982.

Takao, K. and Kikuma, N.: Tamed adaptive antenna array, IEEE Trans. Antennas Propagat., 34, 388-394, 1986.

Takao, K., Fujita, M., and Nishi, T.: An adaptive antenna array under directional constraint, IEEE Trans. Antennas Propagat., 24, 662-669, 1976.

Wang, Y. and Fang, W.: Wavelet-based broadband beamformers with dynamic subband selection, IEICE Trans. Commun., E83B, 819-826, 2000.

Yang, H. and Ingram, M. A.: Design of partially adaptive arrays using the singular-value decomposition, IEEE Trans. Antennas Propagat., 45, 843-850, 1997. 